\title{
EXPERIENCE WITH THE LOW ENERGY DEMONSTRATION ACCELERATOR (LEDA) HALO EXPERIMENT BEAM INSTRUMENTATION*
}

\author{
J. D. Gilpatrick, D. Barr, P. Colestock, L. Day, W. Sellyey, R. B. Shurter, M. Stettler, R. Valdiviez, \\ LANL, Los Alamos, NM 87545, USA \\ M. Gruchalla, J. F. O’Hara, Honeywell Corporation, Los Alamos, NM 87544, USA \\ J. Kamperschroer, M. E. Schulze, General Atomics, Los Alamos, NM, 87544, USA
}

\begin{abstract}
A 52 quadrupole-magnet FODO lattice has been assembled and operated at the Los Alamos National Laboratory. The purpose of this lattice is to provide a platform to measure the resulting beam halo as the first four magnets of the lattice produce various mismatch conditions. These data are then compared with particle simulations so that halo formation mechanisms may be better understood. The lattice is appended to the LEDA $6.7-\mathrm{MeV}$ radio frequency quadrupole (RFQ) and is followed by a short high-energy beam transport (HEBT) that safely dumps the beam into a $670-\mathrm{kW}$ beam stop. Beam diagnostic instruments are interspersed within the lattice and HEBT. The primary instruments for measuring the beam halo are nine interceptive devices that acquire the beam's horizontal and vertical projected particle density distributions out to approximately $10^{5}: 1$ dynamic range. These distributions are acquired using both traditional wire scanners and water-cooled graphite scraping devices. The lattice and HEBT instrumentation set also includes position, bunched-beam current, pulsed current, and beam loss measurements. This paper briefly describes and details the operation of each instrument, compares measured data from the different types of instruments, and refers to other detailed papers.
\end{abstract}

\section{INTRODUCTIONS}

The primary purpose of a 52-quadrupole-magnet FODO lattice is to provide a vehicle to measure phase-space halo. The quadrupole magnets are spaced every $21 \mathrm{~cm}$ so that beam measurement and other mechanical hardware may be interspersed between the magnets. Pictured in Fig. 1 is the lattice that contains beam measurement hardware consisting of a wire scanner and halo-scraper assembly (WS/HS), beam-position monitors, a pulsedbeam-current transformer, and loss monitors [1]. The beam position measurements also have the capability of reporting bunched beam current.

The initial-four quadrupole magnets in the lattice are independently powered so that their gradients are adjusted to match and mismatch the RFQ output beam to the

\footnotetext{
* Work supported by the US DOE, Defense Programs and by the US DOE, Nuclear Energy.
}

lattice. Depending on how the magnets are adjusted, the development of specific types of halos has been documented [2]. These phase space halos manifest themselves as various shapes or "shoulders" on the projected beam distributions as measured by the WS/HS assemblies [3].

Table 1 shows the locations of various beam diagnostic components and steering dipole magnets as defined by the space downstream of the numbered quadrupole magnet. The WS/HS assemblies measure projected beam distributions in both horizontal and vertical planes at 9 locations within the lattice. The first WS/HS located at quadrupole magnet \#4 is used to verify the RFQ-outputbeam distribution. The two sets of four WS/HS provide beam matching and rms beam emittance information in the middle and end of the transport lattice. The set of four WS/HS assemblies located between magnets 20 and 26 measure the beam's match condition after the beam has sufficiently debunched to reduce any longitudinal space charge issues. The set of four WS/HS assemblies located between magnets 45 and 51 provide sufficient mismatch information to detect two possible mismatch modes, the quadrupole and "breathing" modes.

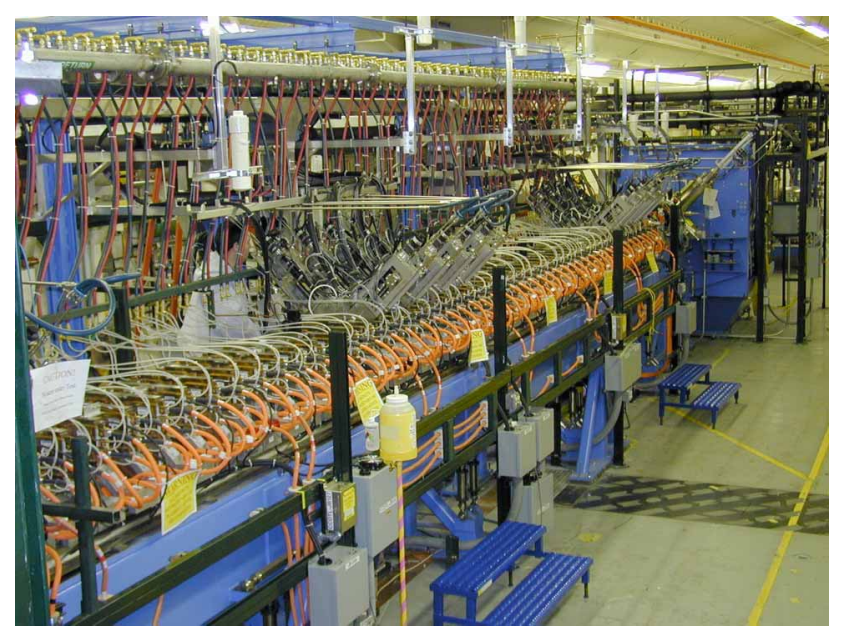

Figure 1. Shown above is the 52 quadrupole magnet lattice with the two clusters of WS/HS beam profile measurements.

The beam-steering plan corrects the beam's position at the end of every set of BPM/associated steering magnet pairs separated by approximately 10 quadrupole magnets 
(see Table 1). Since the FODO-lattice period has a phase advance of approximately 80 degrees, each pair of BPMs can detect, and each pair of steering magnets can correct the beam position and angle.

Pulsed current measurements are monitored at the beginning, middle, and end of the transport lattice. Bunched beam current measurements are acquired at each BPM location. Loss measurements are located over the steering-magnets approximately 1 or $2 \mathrm{~m}$ from the beam line center.

Table 1. Halo Lattice Beamline Component Locations

\begin{tabular}{|c|c|}
\hline Device & Locations (Quadrupole Magnet \#) \\
\hline WS/HS & $4,20,22,24,26,45,47,49,51$ \\
\hline BPM & $5,7,16,18,27,29,37,39,48,50$ \\
\hline Steerers & $3,5,14,16,25,27,35,37,46,48$ \\
\hline BLM & $4,15,26,36,47$ \\
\hline Transformer & $1,23,43$ \\
\hline
\end{tabular}

\section{BEAM PROFILES}

Throughout the halo lattice there are specific beam instruments that acquire horizontally and vertically projected particle-density distributions out to approximately $10^{5}: 1$ dynamic range [4]. We measure the core of the distributions using traditional wire scanners, and the tails of the distribution using water-cooled graphite scraping devices. The wire scanner and halo scrapers (see Fig. 2) are mounted on the same moving frame whose location is controlled with stepper motors [5]. A sequence within the Experimental Physics and Industrial Control System (EPICS) software communicates with a National Instruments LabVIEW virtual instrument to control the motion and location of the scanner/scraper assembly [6]. Both secondary electrons from the wire scanner $0.033-\mathrm{mm}$ carbon wire and protons impinging on the scraper are detected with a lossy-integrator electronic circuit [7]. Algorithms implemented within EPICS and in Research Systems' Interactive Data Language subroutines analyze and plot the acquired distributions.

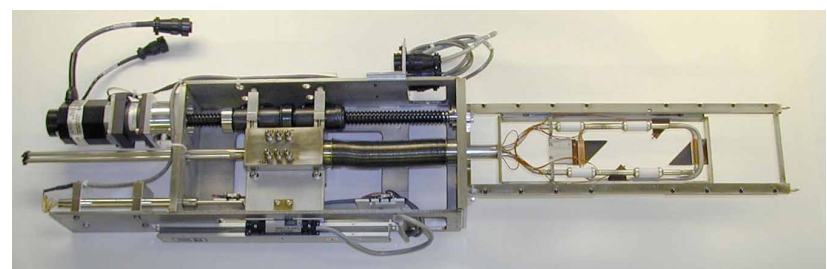

Figure 2. One of two WS/HS actuator assemblies per WS/HS station, these profile measurements have a $10^{5}: 1$ dynamic range.

\section{BEAM LOSS}

Beam loss is measured by detecting the ionizing radiation resulting from the beam intercepting the beam pipe or other structures. A CsI scintillator attached to a 5$\mathrm{cm}$ photomultiplier (PM) [8] allows the detection of the interaction radiation over a dynamic range of $10^{8}: 1$. The signal from high gain PM tube is fed to a transimpedance amplifier via a coaxial cable. The bandwidth of the frontend electronics of $170-\mathrm{kHz}$ allows for observation of $1-\mu \mathrm{s}$ lost beam variations.

Several experiments were performed to validate the performance of the loss monitors. The primary experiment was performed by intentionally allowing 1.6$\mathrm{mA}$ beam current, as measured by the AC current transformers, to impinge on the beam pipe and measuring the resulting signal from the upstream and downstream BLMs. This measurement showed that the BLMs were sufficiently sensitive to detect less than $70 \mathrm{pA}$ of beam loss. However, backgrounds from the beam dump and end of RFQ provide a practical limit of approximately 3 $\mu \mathrm{A}$ of lost beam.

\section{PULSED \& BUNCHED BEAM CURRENT}

The pulsed beam current in the lattice is measured with a set of three AC Current Transformers manufactured for LEDA by Bergoz [1,9]. These transformers performed as specified with resolution and accuracy of $<0.1$ and 0.5 $\mathrm{mA}$, respectively.

The bunched-beam current is the sum of the four electrode signals from a beam position monitor (BPM) [10]. These 350-MHz signals are summed after they have been processed using a logarithmic amplifier/detector. To then acquire a magnitude proportional to the $350-\mathrm{MHz}$ fundamental bunched beam current, the anti-log of this digitized and summed signal is taken. The result of this measurement (shown as open circles) and theory (shown as a solid line) using the simulation code, LINAC, is shown in Fig. 3. Note that as the beam drifts through the halo lattice, the $350-\mathrm{MHz}$ fundamental component decreases in amplitude even

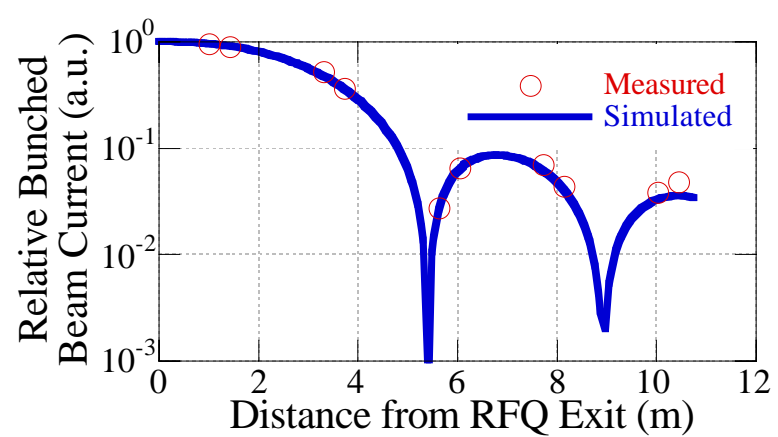

Figure 3. The simulations (line) show a reasonable fit to the measured values of the bunched beam current (circles). The reduction in bunched beam current is due to beam debunching that results in an overlap of itself twice within the halo lattice.

though the pulsed beam current measured by the AC current transformers shows no change. This drop in bunched beam current is due to beam debunching as it drifts through the lattice. Furthermore, Fig. 3 displays how neighboring bunches overlap one another at 2 locations within the lattice. Even though neighboring bunches overlap, there is sufficient $350-\mathrm{MHz}$ current 
modulation to use devices such as BPMs to detect beam position, current, and possibly other beam parameters.

\section{BEAM POSITION}

The beam position measurement consists of a traditional micro-stripline type of beam position monitor (BPM), a cable plant, a $200-\mathrm{kHz} \log$-ratio electronics processor [10], and an interface from LabVIEW to EPICS that provides the linearization and calibration algorithms. Fig. 4 shows the quality of the position and bunched beam current measurements presently installed in the 10 latticeBPM locations. An in-situ calibration procedure corrects for non-linearity and logarithmic non-conformity in the electronics processor [11]. The result of a typical calibration is shown in Fig. 4. Note that the relative position error throughout a 100:1 $(40 \mathrm{~dB})$ dynamic range in beam-current is $<+/-0.03 \mathrm{~mm}$. Furthermore, a dynamic range of 5000:1 (74 dB) is easily obtained using a +/- 0.05-mm criterion.

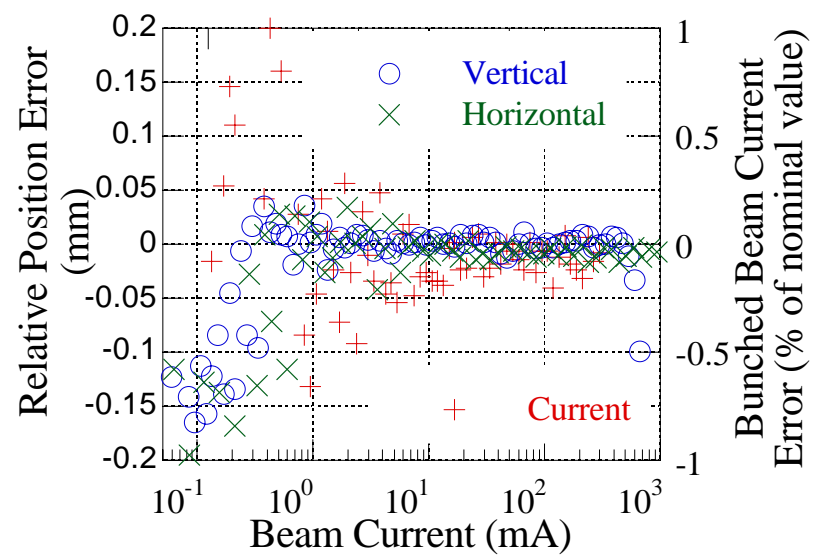

Figure 4. The relative position (circle and $\mathrm{x}$ ) and current errors (pluses) acquired by the in-situ calibration hardware are plotted as a function of beam current. These data are expressed as position error using a $2.32-\mathrm{dB} / \mathrm{mm}$ $\mathrm{BPM}$ sensitivity to a $6.7-\mathrm{MeV}$ proton beam.

For the LEDA halo experiment, absolute beam position error is important to the extent that the beam must be centered within the $28-\mathrm{mm}$ ID beam pipe aperture to within $1 \mathrm{~mm}$. This is especially important since, as the beam is mismatched, the maximum radial extent of the beam has been measured to be $>9 \mathrm{~mm}$ in the areas of the lattice where the beam is nearly round. If the beam is displaced from center too much, scraping occurs and the measured distribution tails are perturbed. Therefore, it was determined that the position measurement must be capable of centering the beam in the beam pipe to within $0.5 \mathrm{~mm}$ (i.e., approximately $1 \%$ of the beam pipe diameter). An error assessment was performed of all known absolute errors, such as mechanical displacement errors of the BPM, cable mismatch errors, errors shown in Fig. 4, etc. The resulting root of the sum of the squares of the known errors is approximately $0.16 \mathrm{~mm}$.

Since the beam position instrumentation also provides bunched beam current, the innate resolution of this measurement is also acquired during the calibration procedure. The resolution of the bunched beam current instrumentation is $<0.75 \%$ of the measured values throughout the range of 1 to $100 \mathrm{~mA}$.

\section{SUMMARY}

During the past six months, we have gained experience measuring the beam halo using instrumentation that includes WS/HS profile, loss, pulsed current, bunched beam current, and beam position measurements. The WS/HS measure the beam's profile with a $10^{5}: 1$ dynamic range. The pulsed and bunched beam current measure these current parameters with a resolution $<0.1 \mathrm{~mA}$. Over a $74 \mathrm{~dB}$ dynamic range in beam current, the position measurements have an accuracy and resolution of $<1 \%$ and $<0.02 \%$ of the beam pipe ID, respectively. These instruments continue to be invaluable for measuring the beam halo so that a clearer understanding of the halo generation mechanisms may be obtained.

\section{REFERENCES}

[1] J. D. Gilpatrick, et al., "Beam Diagnostics Instrumentation for a 6.7-MeV Proton Beam Halo Experiment," LINAC2000, Aug. 21-25, 2000, Monterey, CA, pp. 184-186.

[2] T. Wangler, et al., "Experimental Study of ProtonBeam Halo Induced by Beam Mismatch in LEDA," this conference.

[3] P. L. Colestock, et al., "Measurements of Halo Generation for a Proton Beam in a FODO Channel," this conference.

[4] J. D. Gilpatrick, et al., "Beam-Profile Instrumentation For Beam-Halo Measurement: Overall Description And Operation," this conference.

[5] R. Valdiviez, et al., "The Final Mechanical Design, Fabrication, and Commissioning of a Wire Scanner and Scraper Assembly for Halo-Formation Measurements in a Proton Beam," this conference.

[6] D. Barr, et al., "Design and Experience with the WS/HS Assembly Movement Using LabVIEW VIs, National Instrument Motion Controllers, and Compumotor Electronic Drive Units and Motors," this conference.

[7] M. Gruchalla, et al., "Beam Profile WireScanner/Halo-Scraper Sensor Analog Interface Electronics," this conference.

[8] W. Sellyey, et al., "Experience with Photomultiplier Based Beam Loss Monitors (PMBLM) at the Low Energy Demonstration Accelerator (LEDA)," this conference.

[9] See www.bergoz.com

[10] R. Shurter, et al., "Analog Front-End Electronics for Beam Position Measurement on the Beam Halo Measurement," this conference.

[11] D. Barr, et al., "Upgrade to Initial BPM Electronics Module and Beamline Components for Calibration of the LEDA Beam Position Measurements," this conference. 